M. Pompilii, 2 M. Innamorati ${ }^{2,3}$. G. Giupponi ${ }^{4} \cdot$ R. Pycha ${ }^{5} \cdot$ Z. Rihmer $^{6}$. A. Del Casale ${ }^{1}$. G. Manfredi ${ }^{1}$. A. Celentano ${ }^{1} \cdot$ P. Fiori Nastro ${ }^{7} \cdot$ S. Ferracuti ${ }^{1}$. P. Girardi ${ }^{1} \cdot$ R. Tatarelli ${ }^{1} \cdot$ H.S. Akiskal ${ }^{8,9}$

${ }^{1}$ Department of Neurosciences, Mental Health and Organs Functions, Suicide Prevention Center, Sant'Andrea Hospital, Sapienza, University of Rome, Rome

${ }^{2}$ McLean Hospital, Harvard Medical School, Belmont

${ }^{3}$ Università Europea di Roma, Roma

${ }^{4}$ Ospedale S. Maurizio, Bolzano

${ }^{5}$ Psychiatrischer Dienst Bruneck (BZ), Bruneck

${ }^{6}$ National Institute for Psychiatry and Neurology, Budapest, and Department of Psychiatry and Psychotherapy, Semmelweis, Medical University, Budapest

${ }^{7}$ Department of Psychiatry, Sapienza, University of Rome, Rome

${ }^{8}$ International Mood Center, La Jolla/ CA

${ }^{9}$ San Diego Veterans Administration Medical Center, San Diego

\title{
Erratum zu: Prädiktion des Suizidrisikos bei bipolar und unipolar depressiven Patienten
}

\section{Wechselwirkung von Temperament und Persönlichkeit}

Der Nervenarzt (2009) 80(3):315-323

In diesem Beitrag wurde ein Beitragsautor falsch als „A.D. Casale“ aufgeführt. Richtig muss es lauten: „A. Del Casale“.

Wir bitten dies zu entschuldigen.

\section{Korrespondenzadresse}

Prof. Dr. med. Dr. phil. M. Pompili

Department of Neurosciences, Mental Health and Organs Functions, Suicide Prevention

Center, Sant'Andrea Hospital, Sapienza, University of Rome

Via di Grottarossa 1035-1039, 00189 Rome Italien

maurizio.pompili@uniroma1.it 